\title{
Autonomous Load Balancing Anycast Routing Protocol for Wireless Mesh Networks
}

\author{
Sangsu Jung, Dujeong Lee, Malaz Kserawi, and June-Koo Kevin Rhee \\ Dept. of Information and Communications Engineering and \\ Dept. of Electrical Engineering \\ KAIST, Daejeon, Rep. of Korea \\ \{s.jung,dj.lee, malaz,rhee.jk\}@kaist.ac.kr
}

\begin{abstract}
A wireless mesh network (WMN) is expected to be a key enabler for next generation networking due to its infrastructure-less strengths such as scalability, costefficient rapid deployment, and long distance communications. To boost the strength of WMNs, it is required to balance loads among multiple gateways as well as mesh nodes. However, there have been only few practical and easily-implementable solutions for load balancing of WMNs. In this paper, we propose a distributed autonomous load balancing routing mechanism for anycast WMNs where most traffic flows are for the Internet access through any mesh gateways. Without flooding of control messages, our protocol finds optimized paths implicitly considering geographical distance and a congestion degree based on analogy of physics theory, Poisson's equation and by use of a distributed form of a finite element method. Through simulations, we assess the performance of our protocol compared with the anycast modifications of traditional shortest-path routing and geographic routing under dynamically varying network scenarios.
\end{abstract}

\section{Introduction}

A wireless mesh network (WMN) is one of the next generation ubiquitous network solutions. Because it does not require wired infrastructure, it is cost-efficient, easily deployable, and scalable. Further, it can provide long distance communications through multihop relaying and cover digital-shaded areas to extend communication ranges (e.g. femtocell [1]). To fully utilize the beauty of WMNs, load balancing among multiple mesh gateways (mesh portals) and mesh nodes (mesh points) should be achieved. Because the major purpose of WMNs is to provide the Internet

This work was supported by the Korea Research Foundation Grant funded by the Korean Government (KRF-2008-313-D00685). access through mesh gateways, it is important to control the amount of traffic served by each gateway. One interesting attribute of WMNs is, in its nature, anycast [2] (one-to-one-of-many communication) pattern. In other words, if traffic of a mesh client is served by any possible one gateway, the Internet is accessible. Therefore, conventional shortest-path based unicast (one-to-one communication) routing protocols such as AODV [3] and HWMP [4] are not optimally designed for anycast WMNs because a deterministic single gateway limits the use of resources, otherwise available. Further, when a congested hot spot forms in a path, which causes long delays and frequent packet losses, AODV and HWMP sustain the congestion until new routes are established. In the worst case, a node failure due to a continuous congestion generates the route re-establishment process, which conveys large overheads. In geographic routing such as GPSR [5], congested regions cannot be mitigated, either, because it only considers the proximity to destinations.

As an advanced communication model, we consider anycast routing to efficiently host multiple gateways inspired by physics. Based on an electrostatic potential, the proposed scheme constructs a routing field implicitly reflecting distance to a destination and a degree of congestion in a distributed manner distinctively from HEAT [6] and PB routing [7]. In HEAT, a temperature field is constructed for routing in wireless mesh network. In their model, two values are used for deciding a temperature value. One is the distance from a node to a gateway; the other is the robustness of a path towards a gateway. Because the basic model of it is the Laplace equation, it cannot deal with dynamic network scenarios as stated in Section 2.

On the other hand, a finite difference method (FDM) routing algorithm is suggested in [8] to implement link-diversity routing. It is also based on the temperature field utilizing an FDM, but it is restricted 
to a grid topology; hence another algorithm is required to apply arbitrarily topologies.

In our model, a route is based on a field gradient so that packets are finally forwarded to a node with the lowest gradient. HEAT [6] is a family of anycast routing, but it cannot consider the load distribution of mesh gateways and mesh nodes. Even though PB routing [7] demonstrates the traffic-aware mechanism, our novel approach develops a robust network-wide stable anycast routing model. Notably, our protocol uses only one-hop neighbors' potential information to achieve the global traffic engineering and requires no specific scheduling handler as in back pressure scheduling and routing [9].

In detail, the summary of our work is as follows:

First, we map a network to an electrostatic potential field based on Poisson's equation, which has been verified to be robustly stable in nature as introduced in Section II. When assigned scalar values in the field are maintained, the network is in a steady-state. On the other hand, the scalar value is implicitly determined by proximity to destination and congestion degree.

Second, we deploy a localized algorithm to construct a potential field to solve a global load balancing problem among multiple gateways as well as multiple mesh nodes. Especially, we apply a finite element method (FEM), which is one of numerical analysis techniques, to assign a potential value to each node of an arbitrary mesh network topology in a distributed way. (Section 3)

Third, we propose a novel field based anycast routing [10], which achieves autonomous load balancing against congestion and robustness to node perturbation with few overheads. (Section 4)

Finally, we compare our protocol with shortestpath-based anycast protocol and greedy-forwardingbased anycast protocol in Section 5.

\section{Analogy to Physics: Poisson’s Equation}

The main motivation of our work comes from the essential property of Poisson's equation (1) [11][12]:

$$
\nabla^{2} \phi=-\eta \rho
$$

where $\phi$ presents the potential distribution, $\eta$ is a coefficient, and $\rho$ denotes the charge of a source in physical systems. It is well known that the potential values of physical materials in a steady state must be governed by (1). To design a throughput-optimal and load-balanced stable anycast mesh network, we develop an analogy between electrostatic potential theory and a network routing problem.

We define an undirected graph $G=(N, E)$ where
$N$ is the set of vertices (nodes) and $E$ is the set of edges (links) between nodes in a network. Further, every node $n$ has the set of its one-hop neighbors, $Z(n)$ where $n \in N$. To implement dynamic autonomous traffic load balancing based on (1), we take the source charge, $\rho(n)$, as a packet traffic volume (the number of packets remaining in the queue), and consider $\phi(n)$ as the potential of each node $n$.

For reference, (1) basically states that the potential field is a twice differentiable function; hence the field based on (1) is a monotonously decreasing where $\eta$ and $\rho$ are positive.

Similar approaches are found as Laplace-equationbased routing protocols [6][8]. The Laplace equation (2) is a specific example of (1), where the right hand side is zero with no source dynamics:

$$
\nabla^{2} \phi=0
$$

It governs a thermal system. That is, the temperature distribution in a steady state system converges to a certain value based on (2). However, our distinguishable feature is to deal with a load balancing problem considering source dynamics implying congestion degree observed by queue length, $\rho(n)$.

\section{Distributed Algorithm: Finite Element Method}

To assign the scalar potential value $\phi(n)$ to each node $n$ based on (1), it is required to find an efficient algorithm. As one approach, we derive a distributed form of field calculations with the help of a finite element method (FEM) [11][12] and local equilibrium method (LEM) [13].

For an FEM, each node $n$ constructs triangles with $Z(n): e_{0, n}, \cdots, e_{i, n}, e_{i+1, n}, \cdots, e_{m-1, n}$; which are interpreted as the one-hop neighbor elements to determine $\phi(n)$ where node $n$ has $m$ one-hop neighbors of a geometry model as shown in Fig. 1. Applying a traditional FEM formulation of a Poisson's equation, a primitive solution for a single triangle element $e_{k, n}$ is derived as follows:

$$
\begin{gathered}
\phi(n)\left\|\vec{r}_{k, n}-\vec{r}_{k+1, n}\right\|^{2}=\left(\phi_{k+1, n} \vec{r}_{k, n}-\phi_{k, n} \vec{r}_{k+1, n}\right) \cdot\left(\vec{r}_{k, n}-\vec{r}_{k+1, n}\right) \\
+\eta \Delta_{k} \rho(n)
\end{gathered}
$$

where $\vec{r}_{k, n}$ indicates the real-space distance vector relevant to the conventional notations of $b_{i}$ and $c_{i}$ of [11] and [12]. Further, we define a fractional 


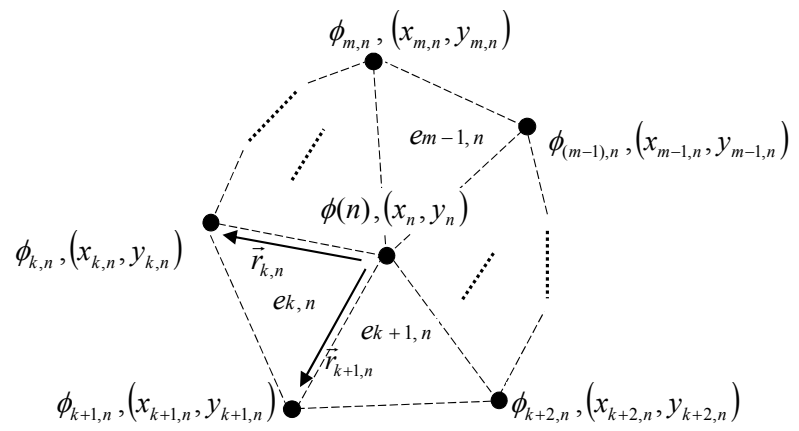

Fig. 1. FEM element geometry model consisting of one hop neighbors.

coefficient $\Delta_{k}$, which represent the portion of the source contribution to element $k$, thus $\sum_{k} \Delta_{k}=1$. Considering the impacts from all neighbor elements, a typical FEM assembles (3) of all neighbors in Fig. 1 into the following form:

$$
\phi(n)=\frac{\sum_{k=0}^{m-1}\left(\phi_{k+1, n} \vec{r}_{k, n}-\phi_{k, n} \vec{r}_{k+1, n}\right) \cdot\left(\vec{r}_{k, n}-\vec{r}_{k+1, n}\right)+\eta \rho(n)}{\sum_{k=0}^{m-1}\left\|\vec{r}_{k, n}-\vec{r}_{k+1, n}\right\|^{2}}
$$

Here, the distance vector comes from the real space, but it can also be substituted by a link cost between neighbors and other metrics.

\section{Autonomous Load-balancing Field- based Anycast Routing Protocol}

Based on Poisson's equation in Section 2 and an FEM in Section 3, we develop Autonomous Loadbalancing Field-based Anycast routing (ALFA) which is a sort of field based routing. In ALFA choosing the forwarding node which has $\arg \min _{z_{l}(n) \in Z(n)} \phi\left(z_{l}(n)\right)$ where $l=0, . ., m-1$, packets are traversed toward the steepest decent and finally arrived at a mesh gateway.

In this section, we illustrate how ALFA initiate and work; summarize several properties of it.

\subsection{Potential Field Creation}

During network bootstrapping, all mesh nodes except mesh gateways are initialized to have zero potential values. To construct a field for routing, it is required to set the boundary condition. In other words, basis points which have the highest value and the lowest value should be defined. Thus, we set the potential of mesh gateways to $\phi(D)=-1,000$ where $D \subset N$ and that of mesh nodes (boundary nodes) which are the set of farthest nodes to a destination to

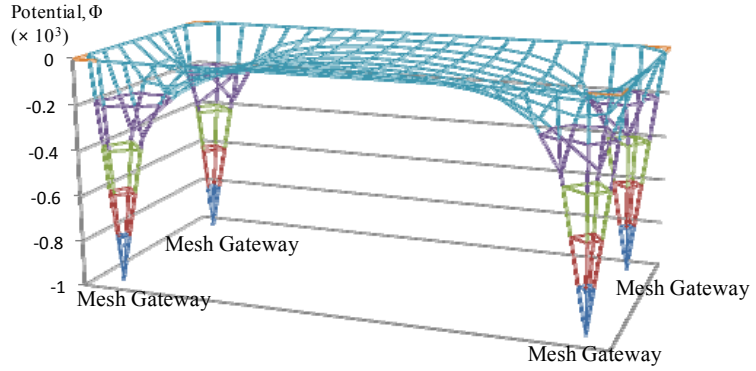

Fig. 2. An example of a potential field in a steady state.

$\phi(B)=0$ where $B \subset N$, to generate a 'field gradient' from mesh nodes to gateways. Due to a pre-defined minimum potential value of mesh gateways, any packet is eventually arrived at one of them. After the initial setting including the boundary condition, each node starts broadcasting its potential value to its onehop neighbors and iteratively re-calculates its value based on (4) for convergence by the local equilibrium method. Within few iterations which spend a very short period of time, all nodes approach $90 \%$ of their steady state in the mean-square-root-error sense. In Fig. 2, we present one example of a potential field in a steady state. When setting a beacon interval to $1 \mathrm{~s}$, usual convergence time takes quite less than $2 \sim 3 \mathrm{~s}$ because every node receives local information several times from multiple neighbors within 1s. After the convergence, 2-3 iterations are required to get the steady-state potentials during packet transmissions.

\subsection{Source Routing for Downlink}

The field of ALFA is for uplink. For downlink, ALFA utilizes source routing. That is, the paths of packets used for uplink are recorded in mesh nodes and mesh gateways; and packets from mesh gateways to mesh nodes are forwarded through recorded paths. This approach maintains the scalability strength of ALFA without additional control overheads. For reference, [14] reports that source routing is an appropriate compromise for downlink routing compared with on-demand routing and proactive routing.

\subsection{Protocol Properties}

The major property of ALFA is autonomous load balancing. For example, when congestion happens at $\mathrm{H}$ as shown in Fig. 3(b), the potential of the node increases so that the potential distribution of the neighbor nodes also increases by (4) from the 


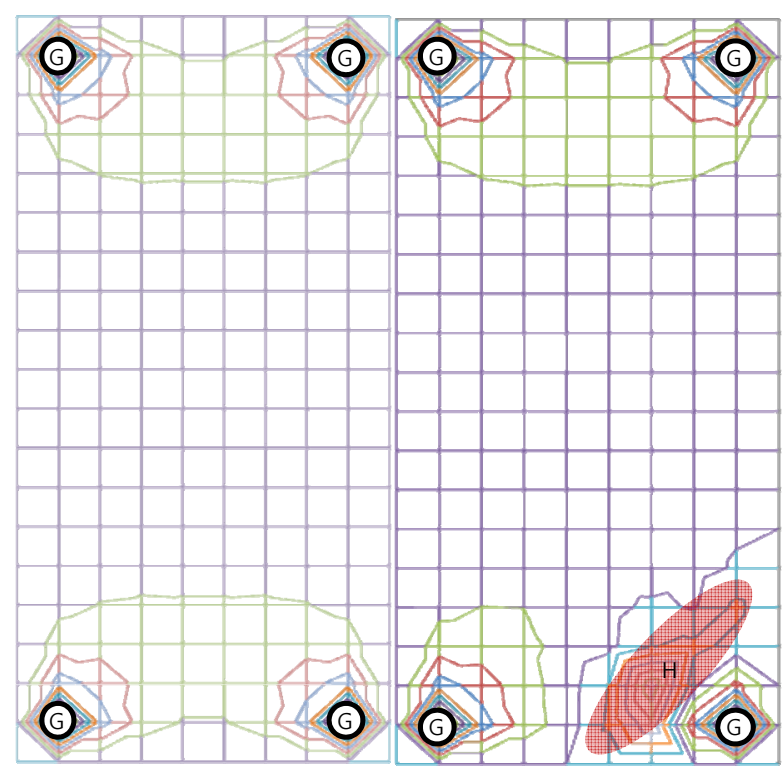

(a)

(b)

Fig. 3. 2D example of potential distribution when there are two mesh gateways; (a) uncongested case, (b) congested case. $\mathrm{G}$ and $\mathrm{H}$ represents for mesh gateways and congested hot spots, respectively.

uncongested potential distribution in Fig. 3(a). Hence, a new routing path is formed based on the changed field, as the traffic tends to avoid the congested high potential area. Here, the local potential adaptation enables packets to be served by another light-weight loaded mesh gateway efficiently utilizing available network resources. Without any centralized controller or handshaking between mesh gateways, ALFA promptly employs mesh gateways depending on situations.

We admit that ALFA can be enhanced with link scheduling modification as in back pressure routing and scheduling. However, the MAC layer of conventional commercial 802.11 devices may not be easily reconfigured and somewhat uneconomical to change the market-deployed products. The beauty of ALFA is to show that network wide traffic engineering at a certain level can be achieved on the network layer.

Another feature of ALFA is the stateless property similar to geographic routing. ALFA continuously provides forwarding with no route re-establishment process learning network-wide routing path. Because ALFA always maintains network-wide field only with local forwarding candidates, it is robust to node failures and scalable.

Because ALFA is a family of field-(gradient)-based routing, it is loop free [7][15]. Furthermore, it also maintains consistency of routing due to the nature of Poisson's equation. Even though it has the stateless property, the potential field does not fluctuate.
Additionally, the potential sensitivity to congestion can be controlled by the choice of a value for $\eta$.

\section{Performance Evaluations}

In this section, we evaluate ALFA using NS-2 [16] incorporating the PHY/MAC models of IEEE 802.11. For simulations, 200 mesh nodes are randomly generated and four mesh gateways are constructed for Internet access under a geographic region of a $2000 \mathrm{~m} \times 4000 \mathrm{~m}$ area. The transmission range of the nodes is set to $250 \mathrm{~m}$. Each data packet (UDP) is 512 bytes long and sent at the rate of 8 to 10 packets per second under the air interface channel bandwidth of $2 \mathrm{Mbit} / \mathrm{s}$. Additionally, $\phi(D)$ for a mesh gateway is assigned to a certain low-enough value (e.g. -1,000).

First, we present the result of comprehensive studies of ALFA for the sensitivity of $\eta$ to load balancing performance; because $\eta$ plays a role to control the impact of congestion degree. Next, we observe the effect of beacon interval to exchange local information on node perturbation cases. Here, the relationship between accuracy and overheads is investigated. Finally, we compare ALFA with shortestpath-based anycast routing (SPR) and geographic greedy-forwarding-based anycast routing (GFR) on the perspective of load balancing and robustness to node failures in dynamically varying network scenarios.

\subsection{Load Balancing vs $\eta$}

We measure the aggregate and individual throughput of mesh gateways to validate a load balancing effect with respect to $\eta$. As $\eta$ increases, the sensitivity to congestion increases, accordingly. For too small $\eta$, ALFA may not be able to avoid congested nodes in the routing path. On the other hand, for too large $\eta$, it may choose an inappropriate path even when the shortest path is lightly congested. As presented in Fig. 4, when $\eta$ is 10,000, the throughput is maximized considering the aforementioned trade-off. More in detail, when $\eta$ is zero, the route cannot adapt to traffic perturbation: One gateway dominantly serves the nodes of a congested hot spot. Because the collision happens more frequently, the packet delivery ratio is lower than the case with $\eta$ of 10,000 . (Fig. 5)

\subsection{Robustness to Node Failure vs Beacon Interval}




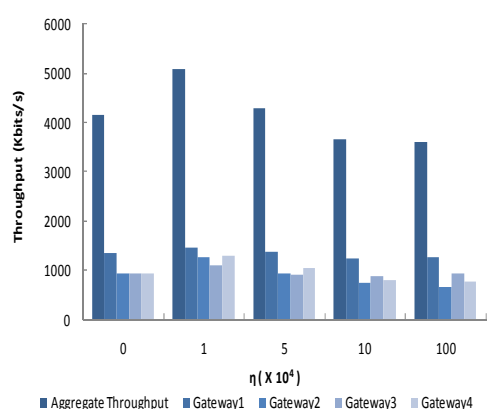

Fig. 4. Throughput w.r.t. $\eta$

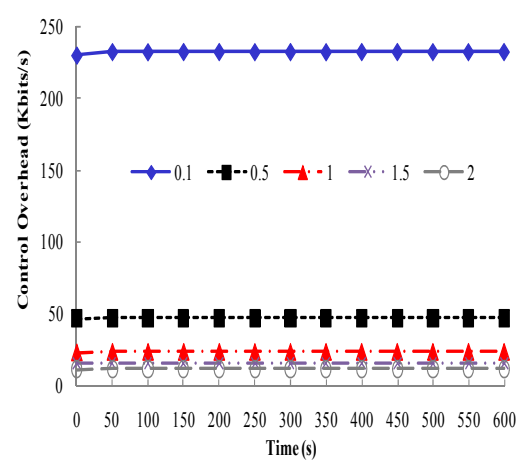

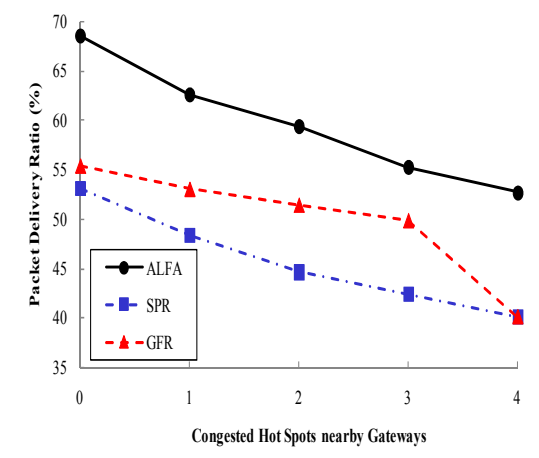

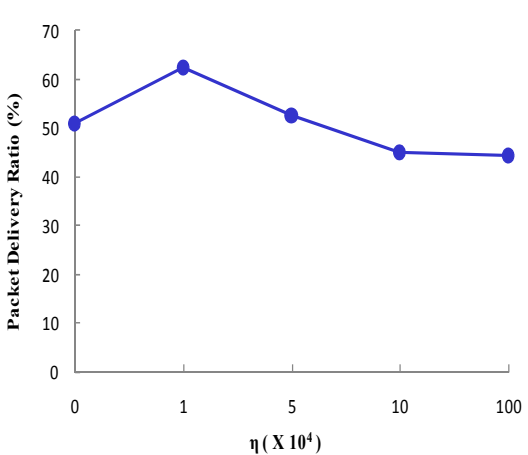

Fig. 5. Packet delivery ratio w.r.t. $\eta$

Fig. 6. Packet delivery ratio w.r.t. beacon interval
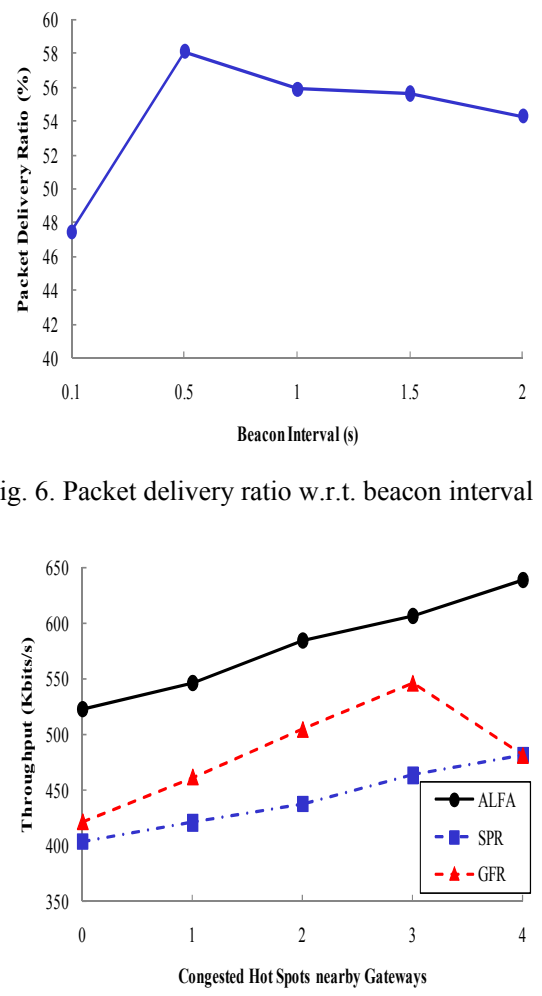

Fig. 7. Control overhead w.r.t. beacon interval Fig. 8. Packet delivery ratio w.r.t. congestion degree Fig. 9. Throughput w.r.t. congestion degree

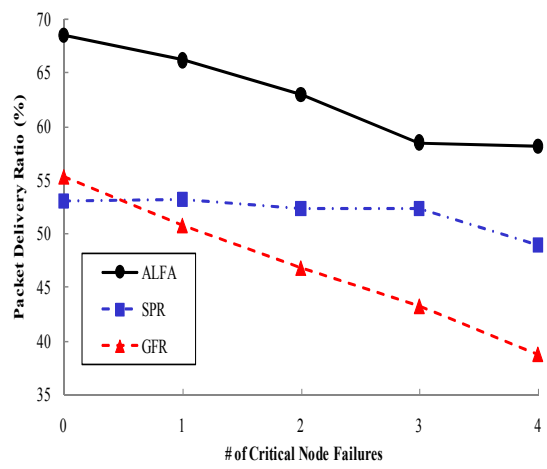

Fig. 10. Packet delivery ratio w.r.t. node failure

To promptly respond to node failures, rapid detection of disappeared nodes is required. One way for this is to set beacon interval short. However, it increases control overheads which degrade the performance of a network. Hence, selecting an appropriate beacon interval is mandatory. We track the packet delivery ratio with respect to beacon interval in node failure scenarios as shown in Fig. 6. At beacon interval of $0.5 \mathrm{~s}$, the performance is the highest. In the case of $0.1 \mathrm{~s}$, the accuracy of information is higher compared to others but control overhead messages occupy critical network resources; hence, it 
the autonomous load balancing effect, we set that up to two hop nodes nearby mesh gateways experience severe congestion increasing congested areas. As presented in Fig. 8, the packet delivery ratio of ALFA is on average $50 \%$ higher than those of SPR and GFR, respectively. Further, the throughput of ALFA continuously and significantly increases; whereas, that of SPR slightly increases and that of GFR suddenly drops as shown in Fig. 9. The reason is that ALFA naturally avoids congested areas; also appropriately diversifies the traffic load of mesh gateways, whereas SPR and GFR maintain current congested paths until new paths are established. Here, ALFA efficiently utilizes mesh gateways until reaching the fundamental limit of whole bandwidth in a network; SPR and GFR spend the available bandwidths of a network.

For the condition of node failures, we dynamically change the frequency of node failures which are critical in paths especially for $150 \sim 350$ s period. In this setting, one to four randomly selected critical nodes repeatedly on and off during the period. Under the conditions, ALFA outperforms around 50 90\% than SPR and GFR in packet delivery ratio (Fig. 10), and $30 \sim 40 \%$ in throughput (Fig. 11); because critical forwarding node failures cause route re-establishment process of SPR and voids in GFR. In Fig. 12, the control overheads of three schemes are presented. At the moment of node failures, the control messages are generated, which cost large network resources. On the other hand, ALFA and GFR show low constant bandwidth share of control messages because they have no route maintenance process. For reference, the reason for the lower overheads of ALFA than that of GFR is that GFR requires the location information of a destination, whereas ALFA does not.

\section{Conclusions}

In this paper, we propose an advanced anycast routing protocol for autonomous load balancing of wireless mesh networks. By an analogy of physics, we develop a potential field-based anycast routing to effectively utilize mesh network capacity. For a distributed protocol, we apply a finite element method (FEM) - a numerical analysis technique - in a localized form to derive a potential for each node by exchanging local potential information only with its one-hop neighbor nodes. Through simulations, we evaluate our protocol with respect to parameters such as $\eta$ and beacon interval; and compare it with anycast modified versions of shortest-path routing and geographic routing. As results, ALFA significantly demonstrates superior performances to both schemes
(30 to 90\% improvement) and ascertains its scalability, autonomous load balancing capability, and robustness to restore from node perturbation for wireless mesh networks. As future work, we will apply our basic framework to other networking areas such as sensor networks, mobile ad hoc networks, and 3GPP LTEAdvanced.

\section{References}

[1] V. Chandrasekhar, J. Andrews, and A. Gatherer, "Femtocell networks: a survey," IEEE Communications Magazine, Vol. 46(9), 2008, pp. 59-67.

[2] C. Metz, "IP anycast point-to-(any) point communication," IEEE Internet Computing, Vol. 6(2), 2002, pp. 94-98.

[3] C. E. Perkins, E. Belding-Royer, and S. R. Das, "Ad hoc on-demand distance vector (AODV) routing," http://www.ietf.org/rfc/rfc3561.txt, July 2003, RFC 3561.

[4] A. Joshi et al., "HWMP Specification," IEEE P802.11 Wireless LANs, document IEEE 802.11-061778r1, Nov. 2006.

[5] B. Karp and H. T. Kung, "GPSR: Greedy Perimeter Stateless Routing for wireless networks," in Proc. of MOBICOM, 2000.

[6] R. Baumann, S. Heimlicher, and B. Plattner, "Routing in large-scale wireless mesh networks using temperature fields," IEEE Network, Vol. 22(1), 2008, pp. 25-31.

[7] A. Basu, A. Lin, and S. Ramanathan, "Routing using potentials: a dynamic traffic-aware routing algorithm," in Proc. of ACM Sigcomm, Karlsruhe, Germany, 2003.

[8] V. Lenders and R. Baumann, "Link-divesity routing: a robust routing paradigm for mobile ad hoc networks," in Proc. of IEEE WCNC, 2008.

[9] L. Tassiulas and A. Ephremides, "Stability properties of constrained queueing systems and scheduling polices of maximum throughput in multihop radio networks," IEEE Trans. on Automatic Control, Vol. 37(12), 1992, pp. 1936-1948.

[10] S. Jung et al., "Distributed Potential Field Based Routing and Autonomous Load Balancing for Wireless Mesh Networks," IEEE Communications Letters, to appear.

[11] L. J. Segerlind, Applied finite element analysis, $2^{\text {nd }}$ edition, Chap. 5, John Wiley\&Sons, 1984.

[12] J. L. Volakis, A. Chatterjee, and L. C. Kempel, Finite element method for electromagnetics, Chap. 4, IEEE Press, 1998.

[13] Z. Li and M. B. Reed, "Convergence analysis for an element-by-element finite element method," Computer Methods in Applied Mechanics and Engineering, Vol. 123, 1995, pp. 33-42.

[14] R. Baumann et al., "Routing packets into wireless mesh networks," in Proc. of IEEE WiMob, 2007.

[15] V. Lenders, M. May, and B. Plattner, "Density-based anycast: a robust routing strategy for wireless ad hoc networks," IEEE/ACM Trans. on Networking, Vol. 16(4), 2008, pp. 852-863.

[16] NS-2, http://www.isi.edu/nsnam/ns/ 